\title{
BMJ Open Cohort profile: the ChicagO Multiethnic Prevention and Surveillance Study (COMPASS)
}

To cite: Aschebrook-Kilfoy B, Kibriya MG, Jasmine F, et al. Cohort profile: the Chicag0 Multiethnic Prevention and Surveillance Study (COMPASS). BMJ Open 2020;10:e038481. doi:10.1136/ bmjopen-2020-038481

- Prepublication history for this paper is available online To view these files, please visit the journal online (http://dx.doi. org/10.1136/bmjopen-2020038481).

Received 23 March 2020 Revised 16 June 2020 Accepted 22 July 2020

Check for updates

(C) Author(s) (or their employer(s)) 2020. Re-use permitted under CC BY-NC. No commercial re-use. See rights and permissions. Published by BMJ.

${ }^{1}$ Department of Public Health Sciences, The University of Chicago, Chicago, Illinois, USA ${ }^{2}$ Institute for Population and Precision Health, The University of Chicago, Chicago, Illinois, USA ${ }^{3}$ Comprehensive Cancer Center, University of Chicago, Chicago, Illinois, USA

${ }^{4}$ Department of Medicine, University of Chicago, Chicago, Illinois, USA

Correspondence to

Dr Briseis Aschebrook-Kilfoy;

brisa@uchicago.edu

\section{ABSTRACT}

Purpose The Chicag0 Multiethnic Prevention and Surveillance Study or 'COMPASS' is a populationbased cohort study with a goal to examine the risk and determinants of cancer and chronic disease. COMPASS aims to address factors causing and/or exacerbating health disparities using a precision health approach by recruiting diverse participants in Chicago, with an emphasis on those historically underrepresented in biomedical research.

Participants Nearly 8000 participants have been recruited from 72 of the 77 Chicago community areas. Enrolment entails the completion of a 1-hour long survey, consenting for past and future medical records from all sources, the collection of clinical and physical measurement data and the on-site collection of biological samples including blood, urine and saliva. Indoor air monitoring data and stool samples are being collected from a subset of participants. On collection, all biological samples are processed and aliquoted within 24 hours before long-term storage and subsequent analysis.

Findings to date The cohort reported an average age of 53.7 years, while $80.5 \%$ identified as African-American, $5.7 \%$ as Hispanic and $47.8 \%$ as men. Over $50 \%$ reported earning less than US $\$ 15000$ yearly, $35 \%$ were obese and $47.8 \%$ were current smokers. Moreover, $38 \%$ self-reported having had a diagnosis of hypertension, while $66.4 \%$ were measured as hypertensive at enrolment.

Future plans We plan to expand recruitment up to 100000 participants from the Chicago metropolitan area in the next decade using a hybrid community and clinic-based recruitment framework that incorporates data collection through mobile medical units. Follow-up data collection from current cohort members will include serial samples, as well as longitudinal health, lifestyle and behavioural assessment. We will supplement self-reported data with electronic medical records, expand the collection of biometrics and biosamples to facilitate increasing digital epidemiological study designs and link to state and/ or national level databases to ascertain outcomes. The results and findings will inform potential opportunities for precision disease prevention and mitigation in Chicago and other urban areas with a diverse population.

Registration NA.
Strengths and limitations of this study

- COMPASS (Chicag0 Multiethnic Prevention and Surveillance Study) has the unique capacity to recruit individuals previously underrepresented in biomedical research.

- Thus far, there is limited variation in socioeconomic strata within race and ethnicity categories as we have oversampled low-income households.

- The prospective cohort design reduces possible recall bias and selection bias.

- The geographically defined cohort design allows for the exploration of an array of environmental and social exposures potentially affecting health outcomes.

- Participants will continue to be recruited and enrolled at medical centres and aboard mobile units, which will allow for the efficient collection of serial biospecimens and tumour tissue as well as comprehensive health history data from electronic medical records.

\section{INTRODUCTION}

Despite the tremendous advances in medicine and public health in recent decades, many areas of the USA have seen a growing gap in health outcomes and health equity. ${ }^{12}$ As one of the most racially segregated cities in the USA, ${ }^{3}$ Chicago reports alarming health disparities and an unequal burden of cancer and chronic risk and mortality, particularly on the South Side. ${ }^{3}$ The South Side of Chicago encompasses roughly $60 \%$ of the city's land area. The South Side is one of the nation's largest contiguous urban AfricanAmerican communities, and is home to a large percentage of the city's 814500 AfricanAmerican residents. ${ }^{4}$ The South Side also includes Chinatown and Bridgeport, the two neighbourhoods with the highest concentrations of Asian-Americans in Illinois, making Chicago the sixth largest Asian-American city in the USA. The most recent census also 
illustrated that Hispanics now account for nearly onethird of the city's total population of 2.7 million. ${ }^{4}$

Chicago faces significant urban challenges, including concentrated poverty, violence and crime, poor housing and living standards, food deserts and environmental exposures such as toxins in the air and water due to waste sites, landfills and abandoned industrial buildings. ${ }^{5-17}$ Despite advances in cancer and chronic disease prevention, screening, diagnosis and treatment that have improved chronic disease risk and outcomes for many Americans, disparities in cancer, cardiovascular disease, diabetes, asthma and maternal child health persist in Chicago. ${ }^{18}$ Disease-related mortality rates reflect continued disparity between non-Hispanic Blacks and Chicagoans of other races. ${ }^{19-22}$ Health disparities are increasingly understood to be a substantial burden on society in terms of healthcare costs, lost productivity and general societal well-being. ${ }^{23}{ }^{24}$ According to the National Institute on Minority and Health Disparities, health disparities are multidimensional, complex phenomena that result from the interaction of multiple contributing factors over time. ${ }^{25}$ These disparities create trajectories of health vulnerability for certain groups of people that vary from the majority norm. ${ }^{26-28}$ Therefore, there is an urgent need to better understand the influence of factors that drive health disparities, such as healthcare coverage and low socioeconomic status. It is also important to identify the still-unknown factors behind health disparities in order to mitigate these trends and reduce the unequal burden of disease.

The University of Chicago is home to the Institute for Population and Precision Health (IPPH), as well as an National Cancer Institute (NCI)-designated comprehensive cancer centre. Given its location in the largest contiguous African-American community in the USA, its extensive community engagement infrastructure and cohort building experience and the availability of innovative and well-established population science resources, we are in a unique position to focus efforts on understudied populations and to study health disparities on Chicago's South Side. To meet the need for a rigorous study of cancer and chronic disease and disparities in the Chicago metropolitan area, we established the ChicagO Multiethnic Prevention and Surveillance Study (COMPASS) in 2013. COMPASS is a population-based longitudinal study of cancer and chronic disease risk and progression with the overall goal of recruiting a diverse study population in Chicago. As those with lowest socioeconomic status and possibly the greatest vulnerability to poor health outcomes have historically been the least likely to enrol in biomedical and biobanking research, ${ }^{29}{ }^{30}$ we have implemented a study design in which recruitment occurs in both the community and the clinic, and involves extensive engagement activities. Because of the growing recognition of health disparities in Chicago, COMPASS data collection efforts have focussed on potentially important factors for minority health and have emphasised recruitment from minority populations.
COHORT DESCRIPTION

\section{Purpose}

The aims and primary scientific goals of COMPASS are to focus on the discovery of cancer and chronic disease in general, with the perspective that social, economic, racial and political marginalisation are often primary contributing factors associated with health disparities. ${ }^{31-41}$ The COMPASS scientific focus areas are illustrated in figure 1. The primary COMPASS aims driving the design are as follows:

1. To discover novel genetic and epigenetic factors related to the risk of cancers among the urban population through comprehensive yet cost-efficient genome-wide screening of germline DNA for single-nucleotide polymorphism and methylation variants. This will be accomplished by efficient typing of case-cohort samples using custom gene-chips based on all novel genetic and epigenetic variants identified through next-generation whole genome deep sequencing and methylation profiling of cohort samples supplemented with previously identified and validated variants from 1000 Genome Project. Similar screening of tumour tissues of cancer cases will also be done to distinguish between inherent and acquired genomic alterations.

2. To elucidate and characterise cancer risk defining germline genetic/epigenetic discoveries, we will prospectively screen baseline serum/plasma and RNA samples from representative samples of matched subcohorts of all four risk-groups (carriers with cancer, non-carriers with cancer, carriers without cancer and non-carriers without cancer) for proteins/hormones/enzymes and messenger RNAs/non-coding RNAs, respectively. Similar screening of tumour tissues of the two cancer groups will also be done to distinguish between inherent and acquired alterations to further elucidate the biology and identification of novel preventive and therapeutic approaches.

3. To integrate the complex interactions of the genomic determinants with non-genomic environmental, nutritional, behavioural and psychosocial determinants to develop a more complete understanding of cancer risk and causation. This includes consideration of the microbiome. This will be accomplished through tailored statistical and causal modelling approaches developed by statisticians and epidemiological methodologists at the University of Chicago.

4. To discover novel environmental (including air, water and household/workplace pollutants) and dietary (including food additives/toxicants and deleterious food items/nutrients) carcinogens that predispose urban populations to a higher risk for various cancers and chronic disease. These discoveries will be made through interdisciplinary efforts involving environmental sensors using nanotechnology and molecular dosimetry for high-throughput screening for biomarkers of exposure to carcinogens, coupled with sophisticated environmental, statistical and systems modelling. 


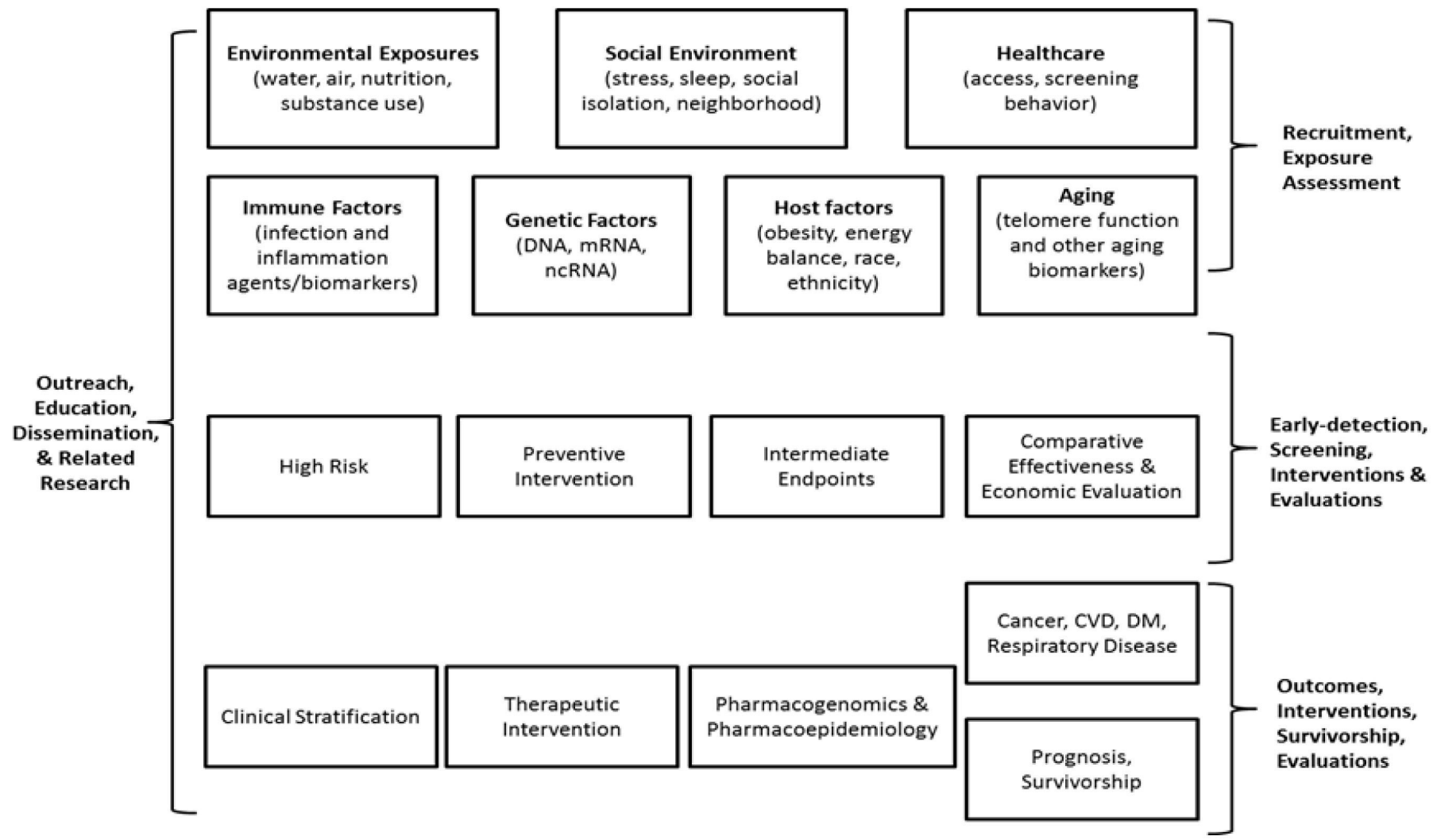

Figure 1 COMPASS scientific focus areas. CVD, cardiovascular disease; DM, diabetes mellitus; mRNA, messenger RNA; ncRNA, non-coding RNA.

5. To identify previously unrecognised factors related to urban lifestyle and behaviour (including stress, energybalance/obesity, sleep and ageing) that increase the risk of various cancers and chronic diseases. These investigations will be conducted through integrated application of behavioural and psychosocial assessment tools, biomarkers of stress/ageing/obesity and novel multilevel and agent-based statistical modelling.

6. To consider new approaches to prevention that are personalised to one's specific risk profile based on their host, environmental/behavioural and genomic profiles by exploiting new knowledge about the riskdriving inherent genomic variants, their interactions with environmental, host, behavioural and psychosocial dynamics, and the downstream molecular alterations in serum/plasma as well as tumour tissues that potentially mediate disease risk. COMPASS will function as a platform for focussed molecular, mechanistic and clinical research.

7. To specifically integrate the diverse data sources named here to investigate opportunities for prevention and early diagnosis, with an emphasis on the mitigation of health disparities.

The recruitment of this diverse cohort presents important opportunities for scientific discovery, disease mitigation, and the alleviation of the dramatic disparities observed in Chicago and other urban centres across the USA. According to the National Cancer Institute, overcoming cancer health disparities is one of the best opportunities for lessening the burden of cancer. ${ }^{42}$ COMPASS data and biosample collection leverages the advanced population science infrastructure at the University of Chicago and preserves the opportunity to use advanced analytical methods and technologies in the future. In the short-term, much will be learnt about the health of Chicago communities. In the long-term, the investigation of the aims above will offer insight into the relative contribution of key health drivers to decrease health inequalities, understand disease risk more generally and elucidate opportunities for prevention and mitigation.

Here, we present this prospective multiethnic cohort study, including a description of the cohort enrolled to date. We also outline the research resources offered by COMPASS and implications for concurrent ancillary studies, as well as future observational and intervention opportunities within the cohort.

\section{Study population}

As of 2019, there were an estimated 2.7 million people and 1.1 million households within the city limits of Chicago. According to a 2018 estimate, the Chicago metropolitan area is the third largest in the USA, with 9.9 million living in city and suburbs combined. More than half the population of the state of Illinois lives in the Chicago metropolitan area. The 2010 US Census shows the population density of the city itself at 11842 people per square mile, making it one of the nation's most densely populated 
cities. The median income for a household in the city as of 2018 was US $\$ 55198$, with $19.5 \%$ of persons in poverty. The goal of COMPASS is to recruit 100000 participants, oversampling the majority minority population (ie, more than $45 \%$ African-Americans and $20 \%$ Hispanics). ${ }^{43}$

COMPASS is a longitudinal cohort study including persons recruited using a population-based approach, a community-based recruitment approach and a hospital/ clinic-based recruitment approach. The multiple recruitment modalities were considered in the early phase of this project in order to capitalise on the strengths and challenges of our Chicago context. As such, here we describe participant recruitment methods using a targeted or community partner approach, as well as at University of Chicago Medicine and on mobile medical units. Parallel data are collected from both community and clinic source populations. These data consist of survey responses, biospecimens including blood, urine, saliva and stool, clinical measures, electronic health records and environmental samples from the home.

To be eligible for participation in COMPASS, participants had to meet the following criteria:

1. Residence in the designated geographical area (census tract).

2. Age 35 and above at the time of contact (to enhance efficiency to obtain cancer outcomes).

3. Ability to complete the consent and interview in English or Spanish.

4. Willingness to provide blood, urine and saliva samples.

5. Provision of a social security number (for outcomes follow-up).

\section{Targeted community-based recruitment}

To explore the feasibility of a population-representative sample for COMPASS, we implemented a recruitment plan to target potentially eligible participants using a twostage cluster sampling approach, first randomising at the census tract level and then identifying potentially eligible persons from within the tract using commercially available address lists. Field staff would then knock on doors and invite the targeted individuals to participate. Specifically, 'cluster sampling' involves dividing the specific population of interest into geographically distinct groups or clusters. Census tract was the primary sampling unit for COMPASS, as census tracts are designed to be homogeneous with respect to population characteristics. In the City of Chicago, there are 798 census tracts; 169 are identified as majority Hispanic, 239 as majority White, 290 as majority African-American and 6 as majority Asian. We identified 120 (15\% of Chicago) study areas of interest using census tracts that would confer the racial/ethnic distribution desired for recruitment. We then used Stata module gsample ${ }^{44}$ to randomly select approximately 120 tracts (40 original census tracts to which 80 tracts have been added) and addresses within blocks. The number of tracts selected was based on an assumption of a $15 \%$ response rate and the assumption that $46 \%$ of residents would be 35 years or older. By sampling at the census tract level, study interviewers would recruit from limited geographical areas where many households could be contacted efficiently, minimising travel time and cost. In particular, we used a probability proportional to size sampling design as this design reduces sampling errors if characters of interest are related to population size. In the first stage, census tracts were randomly selected with probability proportional to the population of census blocks. In the second stage, we randomly chose households within the tract. As two locations within the same census block do not each contribute completely independent information (this is known as the 'intra-cluster correlation'), we selected more clusters rather than more points within any cluster in order to improve precision while maximising study efficiency and minimising cost.

The commercially available address lists used for sampling are available from the US Postal Service's Address Management System and demographic data is available for addresses from commercial sources. The address lists used for COMPASS contained all residential addresses along a mail delivery route, and excluded commercial addresses as well as PO Boxes. The commercial list of addresses provided data for households in the targeted census tracts on age and race and a telephone number (if available). This allowed us to weight the household based on the probability that they would meet eligibility criteria and would reflect the desired racial/ ethnic distribution. We excluded all households that did not appear to have a resident 35 or older.

\section{Engagement for targeted community-based recruitment}

We used the list of addresses in the chosen census blocks to mail a postcard introducing the study and outlining the inclusion/exclusion criteria. The postcard explained that the resident has been selected to participate in COMPASS, what participation would involve, the time commitment, study eligibility criteria, compensation for participation and that multiple eligible household members are welcome to participate. The postcard also provided a time frame in which an interviewer would be requesting participation.

After the postcards were mailed to targeted households, the field interviewers went door-to-door on the targeted study census blocks based on a predetermined schedule to request participation. If a subject did not respond, the interviewer revisited the house within the following 2 weeks. If the interviewer received no response at the third visit, the household would be documented as a nonresponder. By 'dropping in' on all targeted participant homes, a goal was to recruit as least some portion of those individuals at greatest risk of adverse health outcomes.

If the eligible person/s agreed to participate, they enrolled in the study, completed the interview and provided biospecimens and physical assessment data onsite in their home.

The results of the door-to-door recruitment response are presented by Press $e t a l .{ }^{45}$ Taken together, the team 


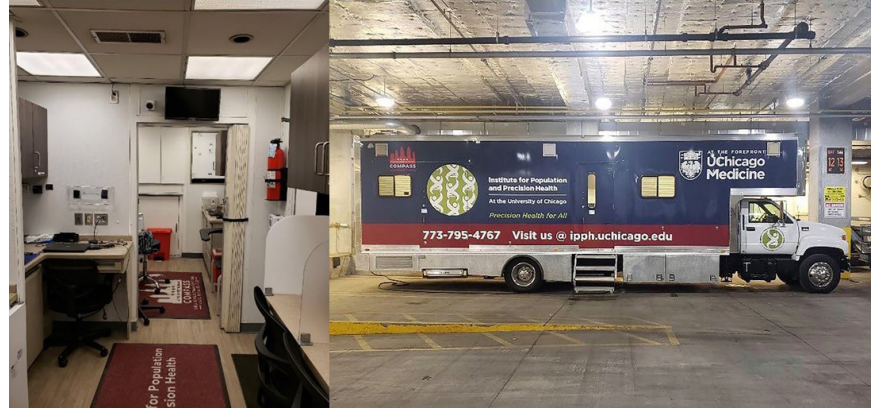

Figure 2 An IPPH mobile unit used for COMPASS enrolment.

achieved a much higher response rate in AfricanAmerican communities than anticipated.

\section{Untargeted community-based recruitment using mobile medical} units

Community-based COMPASS recruitment is supported by two large mobile medical units (figure 2) and the community engagement infrastructure including partner community-based organisations. The mobile medical units have two fully equipped exam rooms with phlebotomy capabilities, a bathroom to facilitate urine collection, are WiFi-enabled and include CLIA (Clinical Laboratory Improvement Amendments) ${ }^{46}$-certified labs with processing/storage capabilities. All study procedures can be accommodated on-board the mobile medical units.

\section{Untargeted community-based engagement}

Untargeted community-based recruitment typically occurs both in conjunction with community partners, as well as without partners in communities targeted for participation. Examples of community partners include faith-based organisations, non-profit organisations, retail stores, civic groups, Chicago Park District facilities and Chicago Public Schools. These partners publicise COMPASS enrolment events and invite participation on days scheduled for mobile unit presence.

\section{Hospital/clinic-based recruitment Study population}

In conjunction with ongoing epidemiologic efforts at University of Chicago Medicine (UCM) administered by the UChicago IPPH (Universityof Chicago IPPH), COMPASS participants are recruited in a UCM research clinic. All UCM patients and their guests aged 35 and older are eligible for participation in COMPASS. In the clinic context, eligibility is not restricted based on place of residence. As such, the eligible study population includes $\sim 60000$ UCM patients per year, over $60 \%$ of whom are African-American. However, this population differs from the community-based sampling in some key ways, and will reflect a lower-risk population from the perspective of cancer health disparities.
Hospital/clinic-based engagement

In-person outreach in the medical centre is conducted by trained research assistants who approach persons in waiting areas and inform them about COMPASS. Those who meet the eligibility criteria are invited to participate. Digital outreach will be expanded in the near future to use email and text introduction. Participants will be able to self-schedule an enrolment and data collection visit at our IPPH research clinic by following a link in the email or text and completing a REDCap (ResearchElectronic Data Capture) scheduling form.

\section{DATA COLLECTION \\ Overview}

Daily enrolment of COMPASS participants was initiated in May of 2013.

\section{Enrolment of cohort members}

The COMPASS interview has been revised and is currently divided into three online modules. These modules are administered via the Qualtrics (Qualtrics, Provo, Utah) survey platform and have been optimised for mobile devices. During their visit to the UChicago research clinic, one of the mobile data collection units, or during community recruitment, participants complete the consent process and survey module \#1 using their own mobile device or an IPPH-provided Apple iPad. Survey modules \#2 and \#3 can be completed onsite during the same visit, or the participant can elect to receive the survey links via email or SMS to complete offsite. COMPASS staff are available onsite to help participants navigate the online consent and survey modules, and participants who choose to complete survey modules \#2 and \#3 offsite are encouraged to reach out to staff via phone or email with any questions or to receive technical assistance.

Once the consent and 60 to $80 \mathrm{~min}$ survey is completed, the following clinical measurements are ascertained: anthropometry, blood pressure and other haemodynamic measures. Study staff measure weight and body fat percentage using a scale and height, hip and waist circumference using a measuring device, and they then measure blood pressure and other haemodynamic parameters using a device that monitors pulse (ie, DynaPulse). An overview of the data collection is provided in table 1. In table 2, the key topics and measurements in the COMPASS questionnaire are outlined. We conducted an extensive review of questionnaires used in other large cancer studies, including the American Cancer Societies Cancer Prevention Study and National Cancer Institute cohorts (including PLCO (Prostate, Lung, Colorectal, and Ovarian Cancer Screening Trial), NIH-AARP and the Agricultural Health Study) in addition to the NHANES (National Health and Nutrition Examination Survey) questionnaires, such that we would be able to harmonise our data in cohort consortium collaboration and maximise our ability to enhance study power for key diseases 
Table 1 An overview of data collection

\begin{tabular}{|c|c|c|c|c|}
\hline Questionnaire data & Blood collection & $\begin{array}{l}\text { Other specimen } \\
\text { data }\end{array}$ & Clinical measurements & $\begin{array}{l}\text { Other data } \\
\text { collection }\end{array}$ \\
\hline Socioeconomic status & $\begin{array}{l}\text { EDTA lavender top } \\
\text { vacutainer }\end{array}$ & Urine collected & Anthropometry & $\begin{array}{l}\text { Electronic medical } \\
\text { records }\end{array}$ \\
\hline Medical history & $\begin{array}{l}\text { SST red tiger top } \\
\text { vacutainer }\end{array}$ & Saliva collected & Blood pressure & Air quality data \\
\hline Psychosocial & $\begin{array}{l}\text { Heparin green top } \\
\text { vacutainer }\end{array}$ & Stool collected & Other haemodynamics & Lead tests \\
\hline Environment & $\begin{array}{l}\text { K2 EDTA blue top } \\
\text { vacutainer }\end{array}$ & & & \\
\hline Lifestyle behaviours & SST gold top vacutainer & & & \\
\hline Family cancer history & Serum red top vacutainer & & & \\
\hline Cancer status & cfDNA collection tube & & & \\
\hline
\end{tabular}

cfDNA, Cell-Free DNA collection tube; SST, Serum-separating tubes.

and populations. Our data dictionary can be found at https://compass.uchicago.edu/.

The purpose of COMPASS anthropometry is to collect body measurements using standardised examination procedures and calibrated equipment. To ensure the collection of high-quality data, COMPASS staff members are trained to follow standardised examination protocols, calibrate equipment according to a prescribed schedule

Table 2 Key topics and measurements in the COMPASS questionnaire

\section{Construct Variable}

Ageing Mother's age at birth; parents' age at death; parents' vital status; osteoporosis

Female medical Age at menarche; breast feeding; fertility interventions; hormonal birth control use; hormone

history replacement therapy; menopausal status; pregnancy history

Lifestyle behaviours Alcohol use; cannabis use; e-cigarettes/vaping; heroin use; lifetime passive smoke exposure; physical activity; self-reported health; smoking cessation; tobacco use history

Follow-up data Address; email address; phone number; contact information of friends or family members; driver's license number; social security number

Immune status Allergies; blood transfusions; influenza immunisation history

Medication use Antihistamine use; anti-hypertensive use; $\mathrm{H} 2$ receptor blocker use; insulin use; non-steroidal antiinflammatory drug use; opioid use; pain status; proton pump inhibitor use; sleep medication use; statin/ cholesterol/lipid lowering drug use

Psychological status Anxiety; depression; stress

Recent medical care Barriers to healthcare access; emergency room/urgent care utilisation; healthcare coverage type and utilisation

duration; healthcare utilisation; screening activities; usual healthcare provider/location

Significant medical Arthritis; asthma; autoimmune disorders; blood cholesterol; dental history; diabetes; family history of history cancer; heart attack/myocardial infarction; hepatitis B; hepatitis C; HIV/AIDs; hypertension; number of children; number of siblings; other chronic diseases; personal history of cancer; sleep apnoea; sexually transmitted diseases; surgeries

Sociodemographic Age; birth date; household income; household size; education level; marital status; occupation; place of status birth; race/ethnicity; sex/gender; work status

Social environment Caregiver status; community social cohesion; night shift work; religion; self-reported patient experience/ satisfaction; sleep habits; stress at home; stress at work

Blood pressure Resting blood pressure; resting heart rate

Body composition Height; hip circumference; waist circumference; weight

Environmental Drinking water sources; lifetime proximity to point sources of pollution; residence during adolescence; exposures self-report crime and violence concerns; self-report environmental concerns; work commute (current and historical) 
and method and precisely measure and record the survey data. Portable digital scales are used in the field on the mobile units by interviewers to measure weight, as well as percentage body fat and water. Body length measurements are made with waist circumference tape and retractable steel measurement tape.

The DynaPulse Pathway System measures central systolic, diastolic and mean arterial blood pressures, and the data are also transmitted to the DynaPulse Analysis Center online for an array of haemodynamic profiles/values. Briefly, DynaPulse, is a cuff-sphygmomanometry-based method, which applies Pulse Dynamics waveform analysis principle and has demonstrated and validated its capability of deriving cardiac output simultaneously with blood pressure, mean arterial pressure, systemic vascular resistance, systemic vascular compliance, brachial artery compliance, distensibility and resistance (brachialartery compliance, brachialartery distensibility and brachialartery resistance), $\mathrm{LV} \mathrm{dP/dtmax} \mathrm{(maximalleft} \mathrm{ventricular} \mathrm{pressure} \mathrm{rise)} \mathrm{and}$ other haemodynamic parameters. These haemodynamic values, collectured for a subset participants, obtained simultaneously allow physicians to correlate the dynamic changes of each parameter to evaluate the physiological conditions of a patient's circulatory system.

Following survey data collection and clinical measurements, COMPASS staff members draws a blood sample. For each participant, the blood sample is collected in cryogenic barcode-labelled tubes as follows: (a) Lavender top EDTA vacutainer $-10 \mathrm{~mL}$; (b) Red and black top SST vacutainer $10 \mathrm{~mL}$; (c) Green top Lithium Heparin vacutainer $-10 \mathrm{~mL}$; (d) Blue top Trace Element K2 EDTA vacutainer - $10 \mathrm{~mL}$; (e) Gold top SST vacutainer - 3.5mL; (f) Red top serum vacutainer $-5 \mathrm{~mL}$; and $(\mathrm{g})$ cfDNA collection tube $-10 \mathrm{~mL}$. The vacutainers are inverted per protocol for proper mixing, ensuring a source of blood material for haematological, biochemical, hormonal, serological and other special tests that require high-quality DNA or RNA.

The blood collection approach was conceived to confer the ability to implement a variety of future analyses relevant for cancer, environmental and population and precision health research. Specifically, the lavender top collection can accommodate assays of whole blood DNA, PBMC (peripheral blood mononuclear cell) - RNA, lymphocyte markers, cytokines, cardiac/cardiovascular disease markers, metabolic panels, cancer panels and investigation of viral and bacterial load (PCR). The green top collection can accommodate assays including thyroid parameters, drug screening and other biochemical tests. The gold top collection accommodates a variety of hormone assays, measurement of lithium, iron, vitamin B12, folate, immunoglobulins, autoantibodies and antibiotic assays. The blue top tubes can accommodate a variety of environmental measurements, including the measurement of some metals.

Study participants are asked to provide a buccal cell sample, using an Oragene DNA self-collection kit, as well as a urine sample.

Participants are eligible to receive three US $\$ 25$ Visa gift cards in recognition of their time. The first gift card is distributed when the participant completes the consent process, survey module \#1, and contributes biospecimens and physical measurement data. The second gift card is given to the participant on completion of survey modules \#2 and \#3. Those who participate in optional home air quality monitoring receive the third gift card when IPPH staff retrieve the air quality devices from their home and the participant completes a short survey on characteristics of their home and environmental exposures.

Study participants are also asked to provide an optional at-home stool sample using a stool sample collection kit. This kit includes instructions to transfer a small swab from toilet paper to a vial. The participant then places the sample vial into a prepaid and addressed envelope and mails it to IPPH.

Biospecimens are stored in medical-grade refrigerators at the IPPH research clinic and aboard the mobile medical units, and are transferred to our laboratory on the University of Chicago campus within 4 to 6 hours of collection.

\section{Biorepository}

At the UChicago IPPH laboratories, the EDTA-mixed blood sample is centrifuged to separate out the plasma sample and aliquoted in multiple $0.5 \mathrm{~mL}$ screw-capped plastic tubes for preservation (to avoid repeated thaw and freeze cycles in future). All components of blood are stored at $-80^{\circ} \mathrm{C}$ and urine samples are stored at $-20^{\circ} \mathrm{C}$ with proper inventory. Freezers are connected to emergency power supply line (red line) and a central alarm system. The saliva samples collected using the Oragene DNA self-collection kit are processed by warming in a hot water bath and stored at $-20^{\circ} \mathrm{C}$ in the IPPH laboratory. Stool samples are snap frozen and stored at $-20^{\circ} \mathrm{C}$.

\section{Environmental sample collection}

Study participants may be asked to allow IPPH staff to collect air quality data from their home using one or more air quality sensors. The sensors provide real-time measures of the presence of particulate matter (PM2.5 and PM10) in addition to other contaminants such as carbonmonoxide, volatileorganic compounds and radon. Air quality data are collected using the COMPASS best practices protocol. Study participants are provided directions to not disturb the sensors and contact the study team with issues. The IPPH staff member schedules a follow-up visit to retrieve the sensors.

Indoor air quality data will be integrated with ambient levels from the Array of Things (AoT) initiative (https:// arrayofthings.github.io/), a joint effort by UChicago and Argonne National Laboratories and supported by the City of Chicago. AoT is currently collecting environmental information on air pollution in addition to light intensity, precipitation, air quality, heat, sound volume and foot traffic through decorative sensors on traffic poles throughout the City of Chicago. The result is a system that collects data about the city at the micro level, including fine-grained pollution levels in different 
neighbourhoods. The diversity of pollutants measured, the number of monitors that will be installed across the city $(>500)$ and the availability of a complimentary sensor for indoor measures, provides an unprecedented opportunity to more accurately assess exposure to air pollution and understand the relative impact of air pollution in Chicago. AoT is placing devices in communities participating in COMPASS. This presents a significant opportunity to understand the role of air pollution in disease aetiology, as well as the potential that findings could inform interventions targeting at-risk Chicago communities.

Details on air quality in-home measurements, ambient concentration modelling and total exposure estimation can be found in accompanying manuscripts. ${ }^{47}$

Interested study participants may be referred to the Chicago Water Quality Study, an initiative of the City of Chicago Department of Water Management. Under this initiative, a water quality kit consisting of sample collection bottles and instructions is mailed to the participant. The participant collects and documents samples per instructions and contacts the Department of Water Management for sample pick up. During the consent process, participants are asked to allow COMPASS to retrieve water quality analysis results from the Department of Water Management.

\section{Data management}

A secure HIPAA-compliant database was created by the Information Technology Group at Dartmouth for the first 4 years of the project. Going forward, all study data is being collected and stored in REDCap and/or Qualtrics (Qualtrics, Provo, Utah). In addition, the University of Chicago Medicine Comprehensive Cancer Center (UCCCC) has created an address database to track the response rate as well as details of non-responders and those who opt not to participate. This database is maintained by the COMPASS informatics team.

\section{Tracking health outcomes}

All participants have provided their social security numbers so that their cancer and vital status can be tracked long-term through the Illinois State Cancer Registry, which is maintained by the Illinois Department of Public Health.

\section{Electronic medical records}

UChicago is a member of the Chicago Area Patient Centered Outcomes Research Network (CAPriCORN) - a platform that integrates EMR across the nine largest academic medical centres in Chicago. COMPASS participants provide consent for access to medical records, which will allow for the efficient collection of comprehensive health history data from EMRs of participants who receive care both at the University of Chicago Medical Centers and outside of our system. The collection of medical records will be completed continuously, when available.

\section{Follow-up data and biosample collection}

Previously enrolled participants will be re-contacted to schedule in-person visits to our IPPH research clinic and our mobile research units. They will be asked to sign an updated consent form and to complete an online follow-up survey in order to understand changes in health status, health behaviours and lifestyle. This visit will also include collection of follow-up biospecimens and physical measurements. Follow-up survey data collection will be requested annually and frequency of in-person biospecimen collection will be funding dependent. In addition, following COMPASS notification that a participant has had a cancer diagnosis, either from the participants themselves or the Illinois State Cancer Registry, we submit a request to the diagnosing and treatment institutions for medical records such that we may ascertain more detailed tumour characteristic and treatment information. We note in the consent that we plan to follow study participants for at least 10 years. In a recent effort to collect follow-up data, we were able to reach $25 \%$ of participants by phone. In the future, we will reach out via text and phone to enhance response rates.

\section{Patient and public involvement}

None of the participants was involved in the questionnaire design, biological measurements or outcome measures; they were likewise not involved in the design, recruitment and implementation of the study. Furthermore, all participants were informed of the use of the data for research in this study. There were no plans to disseminate the study results to participants. When genetic results become available, participants will be contacted and asked if they would like to receive them per the protocol/ consent documents.

All study participants signed a consent form prior to enrolment.

\section{Data analyses}

Baseline data from the cohort were analysed for tabular and descriptive statistics (table 3). Means and SD were calculated for variables with a continuous distribution and proportions were calculated for variables with categorical distributions. Body mass index was computed based on weight and height measured by the interviewers. All analyses were performed using Stata 15 (StataCorp, College Station, Texas) and GeoDa for Windows. ${ }^{48}$

\section{CHARACTERISTICS OF STUDY PARTICIPANTS RECRUITED TO DATE}

Among the 7728 participants enrolled in the study (table 3), 80.5\% identified as African-American, 5.7\% as Hispanic and $47.8 \%$ as men. In addition, over $50 \%$ reported earning less than US\$15000 yearly, $15.2 \%$ reported working full-time, $35 \%$ were obese, $47.8 \%$ were current smokers and $38 \%$ reported having hypertension. High school graduates comprised $19.8 \%$ of the cohort, and $17 \%$ of the participants were married. Consistent 


\begin{tabular}{|c|c|c|}
\hline & Mean \% & $\mathbf{N}$ \\
\hline \multicolumn{3}{|l|}{ Demographics } \\
\hline Average age & 53.7 & 7728 \\
\hline$\%$ Male & 47.8 & 3693 \\
\hline$\%$ Married & 17.4 & 1343 \\
\hline$\%$ African-American & 80.5 & 6224 \\
\hline$\%$ Hispanic & 5.7 & 439 \\
\hline \multicolumn{3}{|l|}{ Lifestyle factors } \\
\hline \% Working full-time & 15.2 & 1174 \\
\hline$\%$ High school graduate/GED & 26.3 & 2030 \\
\hline$\%$ Bachelor's degree or higher & 8.0 & 615 \\
\hline $\begin{array}{l}\% \text { Yearly income less than } \\
\text { US } \$ 15000\end{array}$ & 51.4 & 3975 \\
\hline \% Currently smoke marijuana & 20.2 & 1559 \\
\hline \% Currently smoke cigarettes & 47.8 & 3694 \\
\hline \multicolumn{3}{|l|}{ Medical history (self-report) } \\
\hline$\%$ History of cancer & 5.7 & 441 \\
\hline \% High cholesterol & 24.2 & 1874 \\
\hline \% Type 2 diabetes & 10.1 & 781 \\
\hline \% Hypertension & 38.0 & 2835 \\
\hline \% Health insurance & 76.6 & 5918 \\
\hline$\%$ Heart attack & 4.7 & 367 \\
\hline \multicolumn{3}{|l|}{ Observed values } \\
\hline Average systolic BP (mm Hg) & 136.0 & 6646 \\
\hline Average diastolic BP (mm Hg) & 84.0 & 6216 \\
\hline$\%$ Obese & 35.0 & 2704 \\
\hline
\end{tabular}

BP, blood pressure.

with findings by the Illinois Department of Public Health, the prevalence of diabetes was higher among women than men, and non-Whites compared with Whites. ${ }^{49}$ The overall prevalence of type 2 diabetes in this study was $10.1 \%$.

The maps in figure 3 show the prevalence of the specified variable in the 77 community areas in Chicago. Only community areas with at least 20 observations were included, resulting in 45 communities being analysed. There is an alarming prevalence of high blood pressure, with the majority of the community areas sampled being classified as having stage 1 or 2 hypertension. In addition, the majority of community areas sample have a smoking prevalence of at least $50 \%$.

\section{STRENGTHS AND LIMITATIONS}

COMPASS efficiently recruits individuals previously under-represented in biomedical research. The pilot analysis of the first $8000+$ individuals demonstrates our capacity to recruit such a diverse, urban population. Our UCM research space coupled with our mobile research

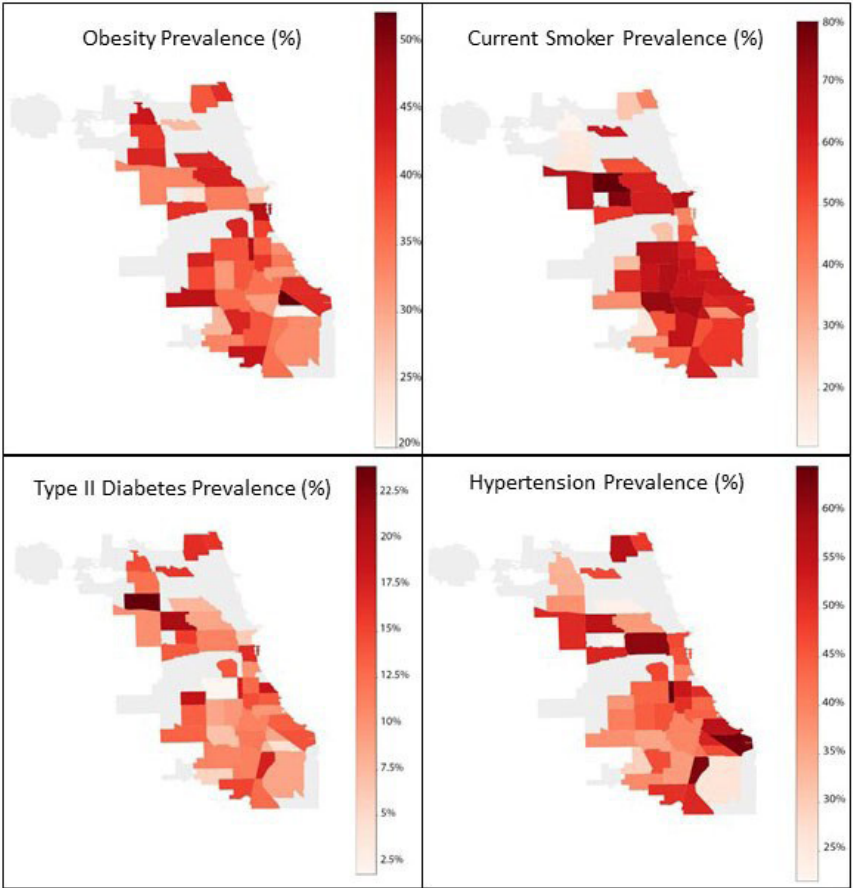

Figure 3 Mapped prevalence of COMPASS self-reported obesity, smoking, type 2 diabetes and hypertension by Chicago community area.

units allow our team to collect and process baseline and follow-up data on campus and in Chicago communities.

Another strength of the study is the prospective cohort design, which reduces possible recall bias and selection bias. Furthermore, the geographically defined cohort design offers a number of advantages for the study of health disparities. The principal reason that we are recruiting a geographical cohort (rather than an occupational cohort, for example) is because it allows for the exploration of an array of exposures potentially affecting health outcomes. In many other cohort designs, subjects are recruited given their unique exposure, such as nurses, farmers, miners and so on. However, the factors that disproportionately affect African-Americans' health status and outcomes in Chicago are largely unknown or under-characterised. Similarly, the relevant risk factors in Hispanic and Asian populations are likely unique and remain under-characterised.

COMPASS is also involved in community engagement efforts to address the needs of the South Side community, through collaboration with the UCCCC Office of Community Engagement and Cancer Health Equity (OCECHE). The overall goals of the OCECHE are to: (1) establish strong partnerships with underserved communities and organisations; (2) support community and local capacity building; and (3) establish sustainable educational programmes. To ensure both academic and community relevance and to help shape future programming and direction, the OCECHE has both a Community and Internal Advisory Board comprising key community and institutional stakeholders. 
In addition, the University of Chicago is a member of the CAPriCORN. CAPriCORN makes it possible for investigators at its partner institutions in Chicago to meet the growing need for the integration of EMRs in epidemiological research, including comprehensive multi-institution tracking/follow-up, such that comprehensive EMRs are available for participants who receive care at multiple institutions. The newly recruited participants will be enrolled through integrated healthcare systems, such as UChicago, which will allow for the efficient collection of serial biospecimens and tumour tissue as well as comprehensive health history data from EMRs. They will be followed longitudinally to study a broad range of risk factors, early markers and outcomes. We will be able to capitalise on the latest advances in the understanding of cancer aetiology, technology for data capture and analytical tools.

The collection of environmental data in COMPASS to understand the role of the environment in health disparities is supported by a new NIEHS (National Institute of Enviornmental Health Sciences)-sponsored environmental health centre in the region-ChicAgo Center for Health and Environment (CACHET). CACHET was established to address EHS (Environmental Health Sciences)-related health disparities as the first centre of its kind in the greater Chicago tri-state area (ie, Illinois, Indiana and Wisconsin). We have developed an environmental monitoring team (used for many NIEHS P30 investigations), infrastructure and expertise to explore the impact of urban environmental exposures that are embedded in COMPASS data collection. The CACHET mission is to elucidate the biological and social underpinnings between relevant urban environmental exposures and human disease and translate the findings to reduce health inequities within our communities. COMPASS is a key cohort synergised by the CACHET Integrated Health Sciences Facility Core at UChicago.

There are some limitations in our study. Thus far, there is limited variation in socioeconomic strata within race and ethnicity categories. Also, the longitudinal nature of the cohort makes it unlikely that we will be able to study cancer-related hypotheses for at least a decade. For rarer diseases, more people and time will be needed. In addition, the oversampling of African-Americans and individuals with low socioeconomic status calls into question the representativeness of the study population. However, given the paucity of cohort studies focussed on this population, COMPASS provides a unique window into the factors impacting health in Chicago. There is also an oversampling of individuals out of work; however, this is possibly also a strength of the study, as some of the highest risk populations not represented in previous works and studies included.

Acknowledgements We thank the dedicated COMPASS field staff and community partners for their support of this work.

Contributors The study protocol was designed by HA, MLi, BA-K, MLe, KHG and $M G K$ and BA-K drafted the manuscript. BA-K and LS oversaw field operations and MGK and FJ oversaw the biosample processing and biobanking. HA, BA-K and KEK oversaw engagement. PZ, RG, AC and ST performed the data quality control and the statistical analysis. All authors participated in the study design, revised the article and approved the final version.

Funding This research was supported by funding from the University of Chicago Medicine Comprehensive Cancer Center and the University of Chicago Institute for Population and Precision Health.

Map disclaimer The depiction of boundaries on this map does not imply the expression of any opinion whatsoever on the part of BMJ (or any member of its group) concerning the legal status of any country, territory, jurisdiction or area or of its authorities. This map is provided without any warranty of any kind, either express or implied.

Competing interests None declared.

Patient and public involvement Patients and/or the public were not involved in the design, or conduct, or reporting, or dissemination plans of this research.

Patient consent for publication Not required.

Ethics approval All study procedures and materials were reviewed and approved by the University of Chicago Biological Sciences Division Institutional Review Board Committee A (approval IRB12-1660).

Provenance and peer review Not commissioned; externally peer reviewed.

Data availability statement Data are available upon reasonable request. COMPASS will continue to collect a rich set of data on multiple exposure domains and health outcomes. For more information, refer to the website: compass. uchicago.edu. Researchers interested in collaboration are invited to propose research questions based on the data available within COMPASS or to submit a request for additional data collection. Requests can be submitted electronically on the COMPASS website and will be reviewed by the COMPASS scientific board. The COMPASS study team is particularly interested in collaborations that will enhance research methods for this type of work, assess the impact of environmental exposure, highlight exposures of key significance in urban communities and address health issues of concern in Chicago and other urban centres.

Open access This is an open access article distributed in accordance with the Creative Commons Attribution Non Commercial (CC BY-NC 4.0) license, which permits others to distribute, remix, adapt, build upon this work non-commercially, and license their derivative works on different terms, provided the original work is properly cited, appropriate credit is given, any changes made indicated, and the use is non-commercial. See: http://creativecommons.org/licenses/by-nc/4.0/.

ORCID iD

Briseis Aschebrook-Kilfoy http://orcid.org/0000-0003-1918-7816

\section{REFERENCES}

1 Meyer P, Yoon P, Kaufmann R. Introduction: CDC health disparities and inequalities report- United States. Center Dis Cont Prevent 2013;62:3-5.

2 Baciu A, Negussie Y, Geller A, et al. Communities in action: pathways to health equity. Washington, DC: National Academies Press, 2017.

3 Orsi JM, Margellos-Anast H, Whitman S. Black-white health disparities in the United States and Chicago: a 15-year progress analysis. Am J Public Health 2010;100:349-56.

4 United States Census Bureau. US census bureau QuickFacts: Chicago City, Illinois. Available: https://www.census.gov/quickfacts/ chicagocityillinois [Accessed 22 Feb 2019].

5 Chicago Department of Public Health. Public health statistics selected public health indicators by Chicago community area. Available: https://data.cityofchicago.org/Health-Human-Services/ Public-Health-Statistics-Selected-public-health-in/iqnk-2tcu/data [Accessed 23 Jan 2020].

6 Chicago Police Department. Crimes - 2001 to present. Available: https://data.cityofchicago.org/Public-Safety/Crimes-2001-topresent-Dashboard/5cd6-ry5g [Accessed 27 Jan 2020].

7 Kolak M, Bradley M, Block DR, et al. Urban foodscape trends: disparities in healthy food access in Chicago, 2007-2014. Health Place 2018;52:231-9.

8 United States Commission on Civil Rights. Food deserts in Chicago: a report of the Illinois advisory committee to the United States commission on civil rights, October 2011. Available: https://www. usccr.gov/pubs/docs/IL-FoodDeserts-2011.pdf [Accessed 23 Jan 2020].

9 Shanahan CE, Spak SN, Martinez A, et al. Inventory of PCBs in Chicago and opportunities for reduction in airborne emissions and human exposure. Environ Sci Technol 2015;49:13878-88. 
10 Jones MR, Diez-Roux AV, Hajat A, et al. Race/ethnicity, residential segregation, and exposure to ambient air pollution: the multiethnic study of atherosclerosis (MESA). Am J Public Health 2014;104:2130-7.

11 United States Environmental Protection Agency. Environmental issues in Southeast Chicago. Available: https://www.epa.gov/il/ environmental-issues-southeast-chicago [Accessed 23 Jan 2020].

12 Sampson RJ, Winter AS. The racial ecology of lead poisoning: toxic inequality in Chicago neighborhoods, 1995-2013. Du Bois Rev 2016;13:261-83.

13 Del Toral MA, Porter A, Schock MR. Detection and evaluation of elevated lead release from service lines: a field study. Environ Sci Technol 2013;47:9300-7.

14 King KE. Chicago residents' perceptions of air quality: objective pollution, the built environment, and neighborhood stigma theory. Popul Environ 2015;37:1-21.

15 Morenoff JD, Sampson RJ. Violent crime and the spatial dynamics of neighborhood transition: Chicago, 1970-1990. Social Forces 1997;76:31-64.

16 Sampson RJ. Chicago and the enduring neighborhood effect. Chicago, IL: University of Chicago Press, 2012.

17 Mohai P, Lantz PM, Morenoff J, et al. Racial and socioeconomic disparities in residential proximity to polluting industrial facilities: evidence from the Americans' changing lives study. Am J Public Health 2009;99:S649-56.

18 Chicago Department of Public Health. Healthy Chicago 2.0 community health assessment. Available: https://www.chicago. gov/content/dam/city/depts/cdph/CDPH/Healthy\%20Chicago/ HealthyChicago_CHA_4102017.pdf [Accessed 11 Jun 2020].

19 Chicago Health Atlas. Cancer deaths. Available: https://www. chicagohealthatlas.org/indicators/cancer-deaths [Accessed 11 Jun 2020].

20 Chicago Health Atlas. Heart disease deaths. Available: https://www. chicagohealthatlas.org/indicators/heart-disease-deaths [Accessed 11 Jun 2020].

21 Chicago Health Atlas. Diabetes - related deaths. Available: https:// www.chicagohealthatlas.org/indicators/diabetes-related-deaths [Accessed 11 Jun 2020].

22 Chicago Health Atlas. Stroke deaths. Available: https://www. chicagohealthatlas.org/indicators/stroke-deaths [Accessed 11 Jun 2020].

23 LaVeist TA, Gaskin D, Richard P. Estimating the economic burden of racial health inequalities in the United States. Int $J$ Health Serv 2011;41:231-8.

24 Thornton RLJ, Glover CM, Cené CW, et al. Evaluating strategies for reducing health disparities by addressing the social determinants of health. Health Aff 2016;35:1416-23.

25 Warnecke RB, Oh A, Breen N, et al. Approaching health disparities from a population perspective: the National Institutes of health centers for population health and health disparities. Am J Public Health 2008;98:1608-15.

26 Agency for Healthcare Research and Quality. 2018 national healthcare quality and disparities report. Available: https://www.ahrq gov/research/findings/nhqrdr/nhqdr18/index.html [Accessed 27 Jan 2020].

27 Adler NE, Stewart J. Health disparities across the lifespan: meaning, methods, and mechanisms: health disparities across the lifespan. Ann New York Acad Sci 2010;1186:5-23.

28 Landrine $\mathrm{H}$, Corral I. Separate and unequal: residential segregation and black health disparities. Ethn Dis 2009;19:179-84.

29 George S, Duran N, Norris K, et al. A systematic review of barriers and facilitators to minority research participation among African Americans, Latinos, Asian Americans, and Pacific Islanders. Am J Public Health 2014;104:e16-31.
30 McDonald JA, Barg FK, Weathers B, et al. Understanding participation by African Americans in cancer genetics research. $J$ Natl Med Assoc 2012;104:324-30.

31 Braveman PA, Kumanyika S, Fielding J, et al. Health disparities and health equity: the issue is justice. Am J Public Health 2011;101:S149-55.

32 Edberg M, Cleary S, Vyas A. A trajectory model for understanding and assessing health disparities in immigrant/refugee communities. $J$ Immigr Minor Health 2011;13:576-84.

33 Edberg M, Cleary SD, Collins E, et al. The safer latinos project: addressing a community ecology underlying Latino youth violence. $J$ Prim Prev 2010;31:247-57.

34 Gaskin DJ, Thorpe RJ, McGinty EE, et al. Disparities in diabetes: the nexus of race, poverty, and place. Am J Public Health 2014;104:2147-55.

35 LaVeist TA, Race ILA. Ethnicity, and health: a public health reader. 2nd edn. San Francisco, CA: Jossey-Bass, 2012: 11-41.

36 Lillie-Blanton M, Lewis CB. Policy challenges and opportunities in closing the racial/ethnic divide in health care. Henry $J$ Kaiser Family Foundation 2005

37 Kawachi I, Daniels N, Robinson DE. Health disparities by race and class: why both matter. Health Aff 2005;24:343-52.

38 United States Census Bureau. Estimates of resident population change and rankings: July 1, 2017 to July 1, 2018 - United States - combined statistical area; and for Puerto Rico. Available: https://factfinder.census.gov/faces/tableservices/jsf/pages/ productview. $x$ html? pid=PEP 2014 PEPANNCHG.US41PR\& prodType=table [Accessed 27 Jan 2020]

39 Smedley BD, Stith AY, Nelson AR, et al. Unequal treatment: confronting racial and ethnic disparities in health care. Washington, DC: National Academies Press, 2003.

40 Starfield B. Global health, equity, and primary care. J Am Board Fam Med 2007;20:511-3.

41 Margellos $\mathrm{H}$, Silva A, Whitman S. Comparison of health status indicators in Chicago: are black-white disparities worsening? Am J Public Health 2004;94:116-21.

42 Silva A, Whitman S, Margellos H, et al. Evaluating Chicago's success in reaching the healthy people 2000 goal of reducing health disparities. Public Health Rep 2001;116:484-94.

43 National Cancer Institute. Cancer disparities. Available: https://www. cancer.gov/aboutcancer/understanding/disparities [Accessed 24 Mar 2019].

44 Jann B. GSAMPLE: Stata module to draw a random sample. statistical software ComponentsS456716, Boston College department of economics, 2005.

45 Press DJ, Aschebrook-Kilfoy B, Lauderdale D, et al. Chicago multiethnic prevention and surveillance study (COMPASS): increased response rates among African American residents in low socioeconomic status neighborhoods. J Racial Ethn Health Disparities 2020. doi:10.1007/s40615-020-00770-2. [Epub ahead of print: 15 Jun 2020]

46 US Food \& Drug Administration. Clinical laboratory improvement amendments (CLIA). Available: https://www.fda.gov/medicaldevices/ deviceregulationandguidance/ivdregulatoryassistance/ucm 124105. htm [Accessed 18 Feb 2019].

47 Aschebrook-Kilfoy B, Zakin P, Craver A, et al. In-home air monitoring in a Chicago multiethnic cohort: preliminary findings Zakin P, Craver A; In preparation.

48 Anselin L, Syabri I, Kho Y. GeoDa: an introduction to spatial data analysis. Geogr Anal 2006;38:5-22.

49 The Illinois diabetes burden. Division of chronic disease prevention and control, office of health promotion, Illinois department of public health, 2012. 